of the endothelium reduces bioavailability of the anti-atherosclerotic signalling radical nitric oxide (NO). We explored the effects of increasing insulin signalling in the endothelium, using novel transgenic mice, over-expressing Type A human Insulin Receptor (HIRECO) in the endothelium, driven by the Tie-2 promoterenhancer.

Methods Semi-quantitative RT-PCT was carried out on various tissues and isolated endothelial cells from lungs to confirm significant levels of human insulin receptor mRNA while the protein expression was confirmed by western blotting on aortic sections or endothelial cells. Lucigenin-enhanced chemiluminescence was exploited to measure superoxide anion levels while; vasomotor functions were assessed in thoracic aortic rings mounted in organ baths.

Results No significant changes in morphological features, metabolic phenotypes or blood pressure regulation were observed between the HIRECO and wild type (WT) littermates. However, plasma insulin levels were similar following an overnight fast, but were decreased in the HIRECO after glucose challenge. HIRECO mice demonstrated significant endothelial dysfunction measured by a blunted response to acetylcholine (Emax, WT vs HIRECO: $84 \pm 3 \%$ vs $68 \pm 3 \%$ respectively; $\mathrm{n}=5, \mathrm{p}>0.05)$. Endothelium-independent response to sodium nitroprusside remained unchanged. The impaired aortic response to acetylcholine was normalised by the specific NADPH oxidase inhibitor peptide, gp91ds-tat, (Emax: $93 \pm 5 \%$; $=6, p<0.05$ ), as well as the superoxide dismutase mimetic, $\mathrm{Mn}$ (III) tetrakis (1-methyl-4-pyrydil) porphyrin pentachloride. Isolated aortic rings of HIRECO exhibited a hypercontractile response to phenylephrine compared to wild type mice (log EC50, WT vs HIRECO: $6.96 \pm 0.03$ vs $7.24 \pm 0.08, \mathrm{n}=6$, $\mathrm{p}<0.01)$. Indeed, HIRECO mice elicited a 1.65-fold increase in the level of superoxide anion production compared to WT. Basal NO bioactivity was decreased in HIRECO compared to WT littermates (Emax upon exposure to eNOS inhibitor, L-NAME in phenylephrineconstricted aorta, WT vs HIRECO: $144 \pm 27.9 \%$ vs $32 \pm 33 \%, n=5$, $\mathrm{p}<0.05)$. However, basal eNOS phosphorylation levels in isolated endothelial cells of HIRECO mice was enhanced 1.56-fold compared to WT littermates.

Conclusions/Implications These data demonstrate enhanced oxidative stress in a novel murine model of increased insulin signalling in the endothelium, leading to reduced bioavailability of nitric oxide and atherosclerosis. These data also demonstrate for the first time, that increased insulin sensitivity in the endothelium, increases the generation of superoxide anion and reduces NO bioavailability.

Funding This work was supported by The British Heart Foundation.

\section{NOX2 NADPH-OXIDASE A NOVEL TARGET TO PREVENT INSULIN RESISTANCE RELATED ENDOTHELIAL CELL DYSFUNCTION}

doi:10.1136/heartjnl-2012-301877b.105

${ }^{1} \mathrm{P}$ Sukumar, ${ }^{* 1} \mathrm{H}$ Viswambharan, ${ }^{1} \mathrm{H}$ Imrie, ${ }^{1} \mathrm{R} \mathrm{M}$ Cubbon, ${ }^{1} \mathrm{~N}$ Yuldasheva, ${ }^{1} \mathrm{M}$ Gage, 'S Galloway, ${ }^{1} \mathrm{~A}$ Skromna, ${ }^{1} \mathrm{~J}$ Smith, ${ }^{1} \mathrm{~K}$ Gatenby, ${ }^{1} \mathrm{D}$ J Beech, ' $\mathrm{S}$ B Wheatcroft, ${ }^{2} \mathrm{~K}$ M Channon, ${ }^{3} \mathrm{~A}$ M Shah, ${ }^{1} \mathrm{M}$ T Kearney. ${ }^{1}$ University of Leeds, Leeds, UK; ${ }^{2}$ University of Oxford, Oxford, UK; ${ }^{3}$ Kings College, London, UK

Introduction Insulin resistance, a central pathophysiological feature of type 2 diabetes is characterised by a deleterious change in endothelial cell phenotype, a hallmark of which is increased generation of reactive oxygen species. We examined the role of NADPH oxidase and specifically NOX2 NADPH oxidase in insulin resistance induced endothelial cell dysfunction. We studied mice with endothelium specific over expression of a dominant negative insulin receptor (ESMIRO) and mice with whole body haploinsufficiency of the insulin receptor $\left(\mathrm{IR}^{+/-}\right)$.
Methods ESMIRO mice, a model of endothelium specific insulin resistance, and $\mathrm{IR}^{+/-}$mice a model of whole body insulin resistance were used to examine the effect of acute and chronic pharmacological inhibition of NADPH oxidase on superoxide production (lucigenin enhanced chemiluminescence) and endothelial function (acetylcholine mediated aortic relaxation). To specifically investigate the role of NOX2, we crossed mice with holoinsufficiency of NOX2 with ESMIRO mice to generate ESMIRO/NOX2 $2^{\mathrm{y}-}$ mice. Data expressed as mean \pm SEM; male mice used for all experiments.

Results Basal superoxide generation in isolated pulmonary endothelial cells (PEC) was increased in both models of insulin resistance (by $130 \%$ in ESMIRO and $220 \%$ in $\mathrm{IR}^{+/-}$compared to wild type, both $\mathrm{p}<0.01 ; \mathrm{n}=3$ for each group). Pre-treating PEC with gp91dstat, a cell permeable specific blocker of NOX subunit assembly and function, reduced the excessive superoxide generation in ESMIRO and $\mathrm{IR}^{+/-}$. Endothelial $\mathrm{NO}$ mediated vasorelaxation in aortic rings from ESMIRO and $\mathrm{IR}^{+/-}$was impaired $(101 \% \pm 11 \%$ relaxation to $1 \mu \mathrm{M}$ acetylcholine in wild type, $61 \% \pm 3 \%$ in ESMIRO $(n=5$, $\mathrm{p}<0.01) ; 91 \% \pm 3 \%$ relaxation in wild type, $75 \% \pm 6 \%$ in $\mathrm{IR}^{+/-}(\mathrm{n}=4$, $\mathrm{p}=0.03)$ ). This was restored by pre-incubating rings with gp91ds-tat ( $92 \% \pm 6 \%$ relaxation in ESMIRO and $93 \% \pm 6 \%$ in $\mathrm{IR}^{+/-}$). Chronic (4 weeks) administration of gp91-ds tat peptide (using osmotic mini-pump) to ESMIRO and $\mathrm{IR}^{+/-}$mice also restored endothelial dependent relaxation (from $83 \% \pm 11 \%$ to $100 \% \pm 9 \%$ in ESMIRO and to $136 \% \pm 11 \%$ in $\left.\mathrm{IR}^{+/-}\right)$. NOX2 gene expression was significantly higher in ESMIRO mice. ESMIRO/NOX2 ${ }^{\mathrm{y} /-}$ mice with complete deletion of NOX2 had significantly greater relaxation responses to acetylcholine than ESMIRO $(77 \% \pm 6 \%$ relaxation in ESMIRO and $100 \% \pm 4 \%$ in ESMIRO/NOX2 ${ }^{\mathrm{y} /-} ; \mathrm{n}=5, \mathrm{p}=0.002$ ). Neither pharmacological nor genetic inhibition of NADPH oxidase had any effect on glucose homeostasis.

Discussion These data in complementary models of insulin resistance demonstrate that acute or chronic pharmacological inhibition of NADPH oxidase reduces superoxide generation and improves endothelial function. Specifically targeting NOX2 also restored endothelial function in ESMIRO mice.

Funding This work was supported by the Medical Research Council UK.

\section{ROLE OF NEURONAL VS ENDOTHELIAL NITRIC OXIDE SYNTHASE IN THE CORONARY BLOOD FLOW RESPONSE TO PACING}

doi:10.1136/heartjnl-2012-301877b.106

${ }^{1} \mathrm{H}$ Shabeeh, ${ }^{* 1} \mathrm{~N}$ Melikian, ${ }^{1} \mathrm{R}$ Dworakowski, ${ }^{2} \mathrm{~B}$ Casadei, ${ }^{1} \mathrm{P}$ Chowienczyk, ${ }^{1} \mathrm{~A}$ M Shah. ${ }^{1}$ King's College London British Heart Foundation Centre of Research Excellence, London, UK; ${ }^{2}$ Department of Cardiovascular Medicine, University of Oxford, Oxford, UK

Background Endothelial nitric oxide synthase (eNOS) has been assumed to be the major source of nitric oxide (NO) regulating human coronary blood flow (CBF). In recent first-in-human studies with a neuronal NOS (nNOS)-selective inhibitor, we provided evidence that nNOS-derived NO regulates basal coronary blood flow whereas eNOS mediates increases in flow in response to the endothelial agonist, substance $\mathrm{P}$. The present study aimed to investigate the effects of nNOS vs eNOS inhibition on coronary blood flow response to increased heart rate.

Methods We studied the effects of the nNOS-selective inhibitor, S-methyl-L-thiocitrulline (SMTC), and the non-selective NOS inhibitor, NG-monomethyl-L-arginine (L-NMMA) at doses previously shown to inhibit nNOS or both $\mathrm{nNOS}$ and eNOS, respectively. 18 patients already undergoing elective cardiac catheterisation for clinical reasons and found to have normal coronary arteries were included. An intracoronary Doppler flow wire was positioned in the coronary artery for measurement of blood flow velocity whereas 